\title{
Двухканальный электронный транспорт в подвешенных квантовых точечных контактах с боковыми затворами
}

\author{
(С) Д.А. Похабов ${ }^{1,2}$, А.Г. Погосов ${ }^{1,2}$, Е.Ю. Жданов ${ }^{1,2}$, А.К. Бакаров ${ }^{1,2}$, А.А. Шкляев ${ }^{1,2}$ \\ ${ }^{1}$ Институт ффизики полупроводников им. А.В. Ржанова Сибирского отделения Российской академии наук, \\ 630090 Новосибирск, Россия \\ ${ }^{2}$ Новосибирский государственный университет, \\ 630090 Новосибирск, Россия \\ E-mail: pokhabov@isp.nsc.ru
}

Поступила в Редакцию 24 августа 2020 г.

В окончательной редакции 26 августа 2020 г.

Принята к публикации 26 августа 2020 г.

\begin{abstract}
Экспериментально изучен кондактанс подвешенного квантового точечного контакта, изготовленного на основе гетероструктур GaAs/AlGaAs с двумерным электронным газом, снабженного боковыми затворами, отделенными от микросужения с помощью литографических траншей. Затворные зависимости кондактанса таких структур соответствуют необычному двухканальному режиму с независимым квантованием кондактанса каналов: кондактансом отдельных каналов можно независимо управлять с помощью двух боковых затворов. Рассмотрен электростатический механизм образования двухканальной структуры внутри одиночного сужения, связанный с латеральным перераспределением низкоподвижных $X$-долинных электронов, содержащихся в сверхрешеточных слоях гетероструктуры, приводящий к образованию в средине квантового точечного контакта потенциального барьера, разделяющего электроны проводимости на два канала, симметрично разнесенные к литографическим траншеям, задающим геометрию наноструктуры.
\end{abstract}

Ключевые слова: квантовый точечный контакт, подвешенные полупроводниковые наноструктуры, мультиканальный транспорт, квантование кондактанса.

DOI: $10.21883 /$ FTP.2020.12.50235.9512

\section{1. Введение}

Квантовый точечный контакт (КТК), как правило, представляет собой узкое микросужение в двумерном электронном газе, ширина которого сравнима с фермиевской длиной волны электрона, а длина много меньше длины свободного пробега электрона. Такое микросужение может быть создано, например, приложением обедняющего напряжения на расщепленный металлический затвор или с помощью литографических траншей, отделяющих канал КТК от боковых затворов. Типичной зависимостью кондактанса от затворного напряжения является ступенчатая кривая с плато при значениях кондактанса $2 e^{2} / h[1,2]$.

В отличие от КТК с расщепленным затвором, где затвор формирует ограничивающий потенциал и одновременно управляет им, в КТК с боковыми затворами канал формируется литографически посредством траншей, что расширяет возможности использования боковых затворов для модификации ограничивающего потенциала, позволяя использовать их практически независимо и прикладывать довольно большие разности потенциалов между ними (до 20 В). Ранее было показано, что приложение разности напряжений между боковыми затворами позволяет создавать латеральные электрические поля, достаточные, чтобы индуцировать спиновую поляризацию в КТК на основе как InAs [3], так и GaAs [4].

Изучение электронного транспорта в одиночных КТК с боковыми затворами, отделенными от микросужения с помощью литографических траншей, показало, что вблизи краев микросужения, вдоль литографических траншей, могут формироваться дополнительные проводящие каналы $[5,6]$. Природа их возникновения до конца не ясна и, по-видимому, заключается в специфических электростатических условиях, реализующихся в КТК в результате вытравливания траншей.

Интересная возможность изменить электростатические условия заключается в том, чтобы подвесить КТК путем его отрыва от подложки с высокой диэлектрической проницаемостью. Подвешивание создает условия для усиления кулоновского взаимодействия как между электронами проводимости, так и между электронами и окружающими их заряженными примесями. Усиление межэлектронного взаимодействия подтверждается экспериментами с подвешенными наноструктурами [7-10]. Кроме этого было показано, что электрон-электронное взаимодействие в тонких пленках может иметь качественные отличия, проявляющиеся в притяжении и спаривании электронов [11-13]. Особенностью подвешенных наноструктур является также отсутствие отвода тепла непосредственно через подложку и связанный с этим характер явлений теплопереноса $[14,15]$.

Двухканальный транспорт наблюдали ранее в вертикально совмещенных спаренных КТК, созданных на основе гетероструктур с двойными квантовыми ямами $[16,17]$. Двухканальный режим был также реализован и в одиночном КТК в условиях низкой концентрации электронов и слабого ограничивающего потен- 


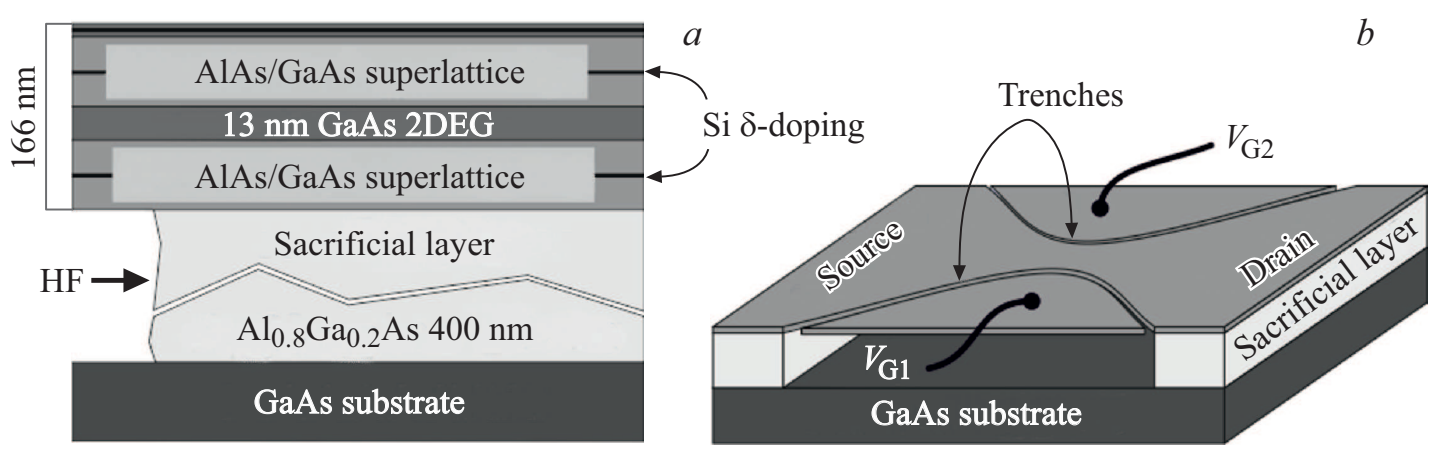

Рис. 1. Схематические изображения: $a$ - гетероструктура GaAs/AlGaAs с жертвенным слоем и двумерным электронным газом, $b-$ подвешенный квантовый точечный контакт с боковыми затворами.

циала [18-21]. В таких условиях электрон-электронное взаимодействие может приводить к вигнеровской кристаллизации. Известно также, что при ослаблении ограничивающего потенциала или увеличении концентрации электронов в вигнеровском кристалле происходит фазовый переход „струна-зигзаг“ [22,23], который регистрируется в измерениях кондактанса в виде удвоения ступеней квантования кондактанса. Формирование двухрядного транспорта было наглядно показано в экспериментах по поперечной магнитной электронной фокусировке [24,25]. Однако в перечисленных работах речь не идет о независимом квантовании кондактанса отдельных каналов. Напротив, в этих работах подчеркивается сильная корреляция между электронами в двух каналах. Таким образом, на данный момент мультиканальный транспорт в КТК с независимым квантованием кондактанса является слабо изученным.

В настоящей статье мы сообщаем о наблюдении двухканального электронного транспорта в подвешенном, т.е. оторванном от подложки, КТК на основе гетероструктур $\mathrm{GaAs} / \mathrm{AlGaAs}$, управляемом контролируемым образом двумя боковыми затворами. Мы показываем, что кондактансом этих каналов можно управлять независимо. В работе приводятся также результаты измерений зависимости кондактанса от напряжения исток-сток для двух отдельных каналов. Такие измерения позволяют определить межподзонные расстояния в двух отдельных каналах. Кроме того, мы рассматриваем электростатический механизм формирования двухканальной структуры КТК, основанный на перераспределении зарядов в гетероструктуре в результате вытравливания литографических траншей и удаления слоев, содержащих доноры.

\section{2. Методика эксперимента}

В эксперименте исследовался электронный транспорт в КТК на основе гетероструктур с жертвенным слоем, позволяющих создавать как не подвешенные, так и подвешенные образцы. Экспериментальные образцы изготавливались на основе гетероструктур $\mathrm{GaAs} / \mathrm{AlGaAs}$ с двумерным электронным газом (ДЭГ/2DEG), выра- щенных методом молекулярно-лучевой эпитаксии на подложках $\mathrm{GaAs}(001)$. Сначала на подложке выращивался жертвенный слой $\mathrm{Al}_{0.8} \mathrm{Ga}_{0.2} \mathrm{As}$ толщиной 400 нм, а поверх него выращивалась сверхрешеточная структура $\mathrm{GaAs} / \mathrm{AlAs}$ толщиной 166 нм, содержащая ДЭГ в слое GaAs толщиной 13 нм, залегающем на глубине 90 нм от поверхности и представляющем собой прямоугольную симметричную квантовую яму для электронов. Подвижность и концентрация электронов составляли $2 \cdot 10^{6} \mathrm{~cm}^{2} /(\mathrm{B} \cdot \mathrm{c})$ и $(6-7) \cdot 10^{11} \mathrm{~cm}^{-2}$ соответственно при температуре $4.2 \mathrm{~K}$. Квантовая яма с высокоподвижным ДЭГ окружена сверхрешеточными слоями, содержащими $\delta$-слои донорной примеси кремния на расстоянии 30 нм по обе стороны от ямы и низкоподвижные $X$-долинные электроны, не участвующие в проводимости, но экранирующие флуктуации примесного потенциала, приводя к увеличению подвижности в ДЭГ. Концепция использования низкоподвижных $X$-долинных электронов в гетероструктурах $\mathrm{GaAs} / \mathrm{AlGaAs}$ изложена в работе [26] и в настоящее время широко используется для создания высокоподвижного ДЭГ. Существование $X$-долинных электронов подтверждается исследованиями вольт-фарадных характеристик и спектрами фотолюминесценции, а также самосогласованными расчетами зонной структуры в соответствующих гетероструктурах со сверхрешетками [26]. Схематическое изображение гетероструктуры приведено на рис. 1, $a$. Экспериментальные образцы КТК представляли собой адиабатические сужения шириной 800-900 нм, снабженные боковыми затворами, отделенными от проводящего канала траншеями шириной 100-150 нм. Боковые траншеи были изготовлены посредством электронной нанолитографии с последующим анизотропным реактивным ионным травлением в плазме $\mathrm{BCl}_{3}$. Для литографии использовался электронный резист полиметилметакрилат (ПММА) толщиной $\sim 200$ нм. Резист экспонировали в пучке электронов с энергией 20 кэВ. Образцы подвешивались путем селективного вытравливания жертвенного слоя из-под наноструктуры с помощью водного раствора плавиковой кислоты. Подвешенный КТК схематически изображен на рис. $1, b$. Образцы были снабжены омиче- 
скими контактами $\mathrm{Au} / \mathrm{Ni} / \mathrm{Ge}$, позволяющими проводить стандартные измерения кондактанса по четырехточечной схеме. Измерения проводились при температуре $4.2 \mathrm{~K}$ с помощью техники синхронного детектирования в режиме линейного отклика при приложении переменного напряжения $V_{\mathrm{AC}}$ амплитудой 30 мкВ с частотой 70 Гц между истоком и стоком. Кондактанс $G=I_{\mathrm{AC}} / V_{\mathrm{AC}}$ измерялся как функция напряжений $V_{\mathrm{G} 1}$ и $V_{\mathrm{G} 2}$, приложенных на затворы, а также напряжения исток-сток $V_{\mathrm{SD}}$.

\section{3. Экспериментальные результаты}

На рис. 2 представлен кондактанс подвешенного КТК как функция суммы синхронно изменяющихся напряжений на боковых затворах при различных значениях разности затворных напряжений в диапазоне от -13 до +13 В. На экспериментальных кривых отчетливо видны плато при значениях кондактанса, кратных $2 e^{2} / h$. Однако кривые имеют не просто ступенчатый, а более сложный вид, характерный для спаренных КТК [16,17]. На кривых, соответствующих значениям разности затворных напряжений $\sim(-13) \mathrm{B}$, можно наблюдать плато кондактанса при значении $0.5\left(2 e^{2} / h\right)$, возникновение которого может быть связано с эффектом латерального спин-орбитального взаимодействия [27,28].

На рис. 3 представлена зависимость транскондактанса, т.е. производной кондактанса $G$ по затворному напряжению, $d G / d\left(V_{\mathrm{G} 1}+V_{\mathrm{G} 2}\right)$, подвешенного КТК от суммы и разности затворных напряжений. Величина транскондактанса показана на рисунке оттенками серого: более темные области соответствуют плато кондактанca, а светлые - переходам между ними. Приведены значения кондактанса в единицах $2 e^{2} / h$. Наблюдаемая картина не типична для одноканального электронного транспорта, для него характерна картина чередующихся параллельных полос. Отчетливо видно несколько по-

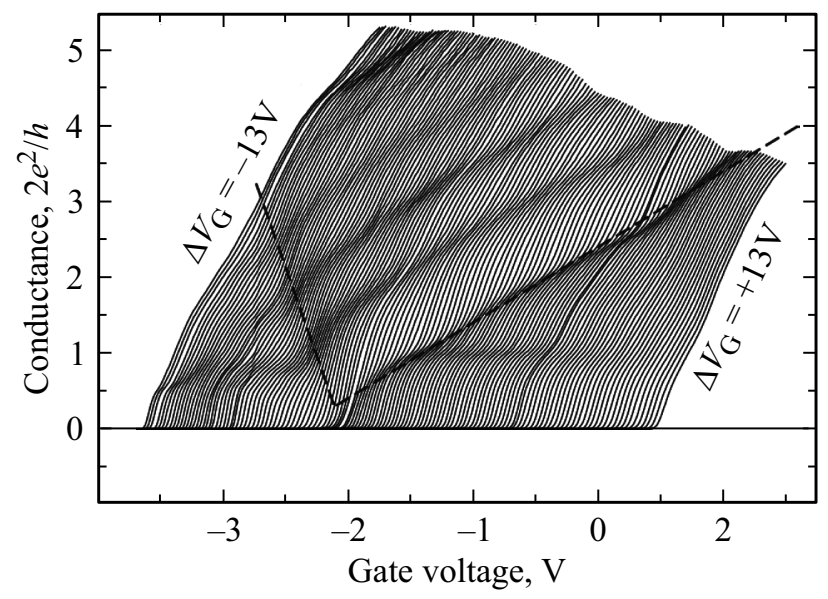

Рис. 2. Зависимость кондактанса подвешенного квантового точечного контакта от суммы затворных напряжений. Разные кривые соответствуют различным значениям разности затворных напряжений $\Delta V_{\mathrm{G}}$ в диапазоне значений от -13 до $+13 \mathrm{~B}$.

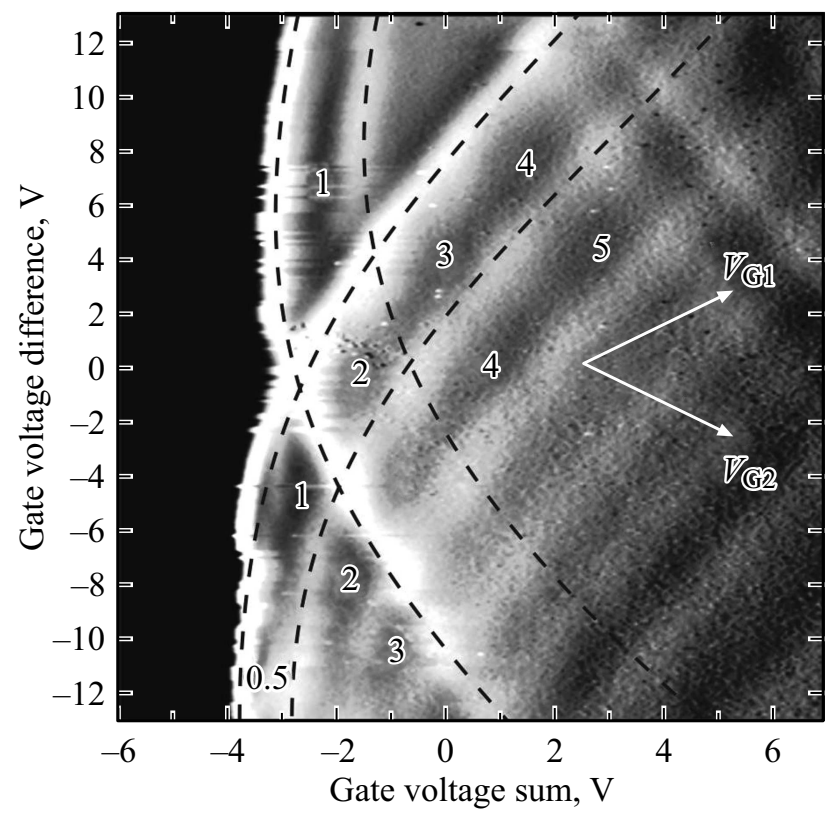

Рис. 3. Зависимость транскондактанса $d G / d\left(V_{\mathrm{G} 1}+V_{\mathrm{G} 2}\right)$ подвешенного квантового точечного контакта от суммы $V_{\mathrm{G} 1}+V_{\mathrm{G} 2}$ и разности $V_{\mathrm{G} 1}-V_{\mathrm{G} 2}$ затворных напряжений. Темные области соответствуют плато квантования кондактанса. Приведены значения кондактанса в единицах $2 e^{2} / h$.

чти параллельных линий, причем можно выделить два семейства линий. Примеры таких линий, разделяющих области плато, показаны на рис. 3 (штриховые линии). Наблюдаемое поведение можно объяснить формированием двух параллельных проводящих каналов. При этом кондактанс каждого канала образует картину параллельных наклонных чередующихся полос. Их суммарный кондактанс, измеряемый в эксперименте, выглядит как наложение этих двух картин.

На рис. 3 можно мысленно выделить три характерных области: две области существования одного из двух каналов, наблюдающиеся при больших абсолютных значениях разности затворных напряжений, и область их сосуществования. На рис. 2 эти три области отделены друг от друга штриховыми линиями. В одноканальных областях линии, разделяющие плато, почти вертикальны, что указывает на симметричное расположение проводящего канала относительно двух боковых затворов. Действительно, в одноканальной области кондактанс канала управляется преимущественно суммой затворных напряжений и практически не зависит от их разности. Другими словами, затворы почти одинаково влияют на кондактанс канала, что указывает на равноудаленность канала от боковых затворов.

В двухканальном режиме, наблюдающемся в области малых абсолютных значений разности затворных напряжений, кондактанс каждого из двух каналов управляется некоторой линейной комбинацией затворных напряжений $\left(\alpha V_{\mathrm{G} 1}+\beta V_{\mathrm{G} 2}\right)$, где коэффициенты $\alpha$ и $\beta$ пропорциональны емкостным коэффициентам „затвор-канал“. 

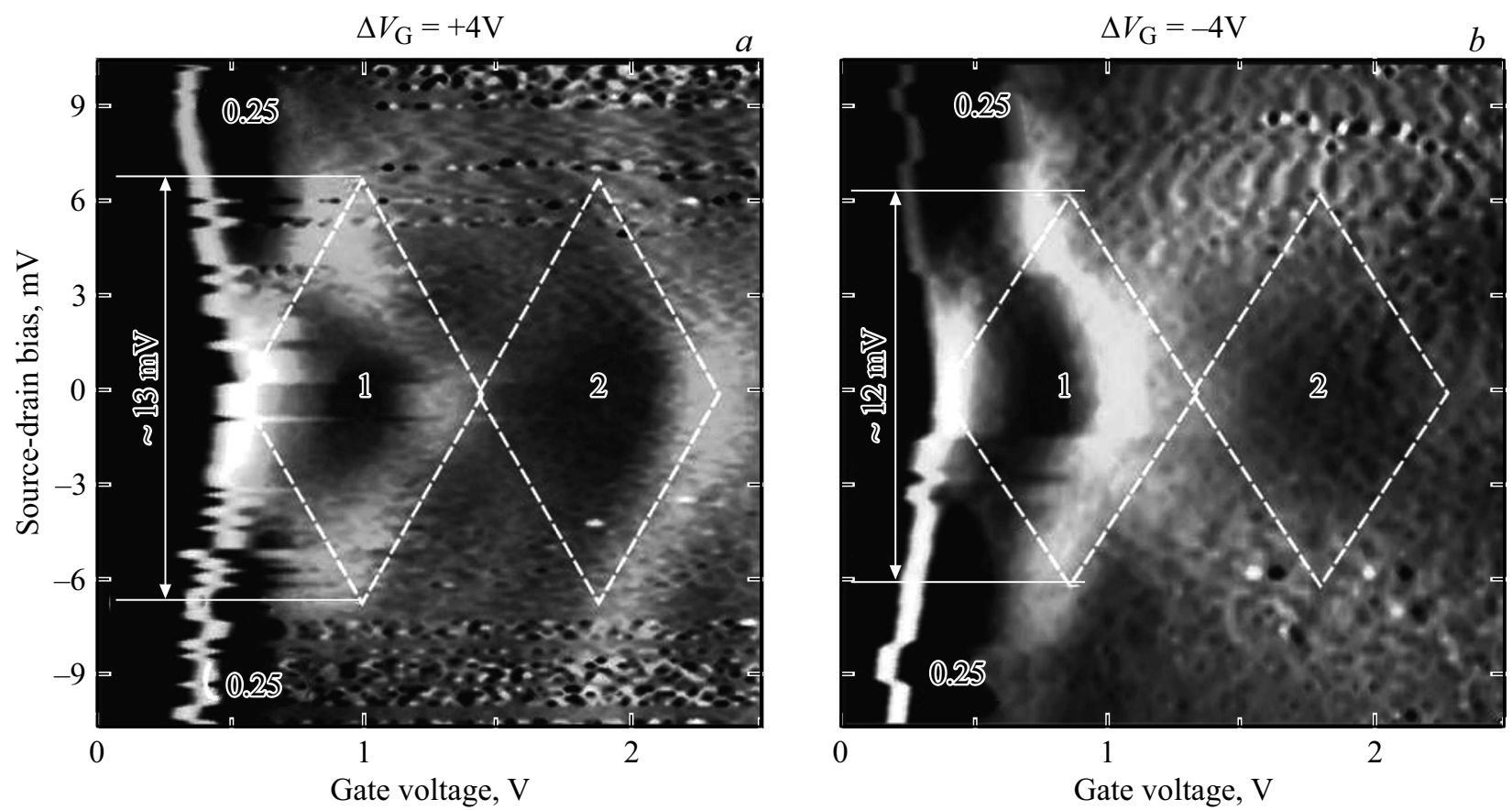

Рис. 4. Зависимость транскондактанса $d G / d\left(V_{\mathrm{G} 1}+V_{\mathrm{G} 2}\right)$ подвешенного квантового точечного контакта от суммы $V_{\mathrm{G} 1}+V_{\mathrm{G} 2}$ затворных напряжений и напряжения между истоком и стоком $V_{\mathrm{SD}}$ при разности $V_{\mathrm{G} 1}-V_{\mathrm{G} 2}$ затворных напряжений $-4(a)$ и $+4 \mathrm{~B}(b)$. Темные области соответствуют плато квантования кондактанса. Приведены величины кондактанса в единицах $2 e^{2} / h$.

При этом, если кондактанс одного из них управляется линейной комбинацией $\left(\alpha V_{\mathrm{G} 1}+\beta V_{\mathrm{G} 2}\right)$, то другого $\left(\beta V_{\mathrm{G} 1}+\alpha V_{\mathrm{G} 2}\right)$, что указывает на симметрию в расположении двух каналов относительно середины КТК. Из эксперимента следует отношение $\alpha / \beta \approx 3$. Таким образом, ближний к каналу боковой затвор влияет на кондактанс канала в $~ 3$ раза сильнее, чем дальний. Отметим, что, изменяя затворные напряжения в указанной комбинации, мы можем независимо управлять кондактансом отдельных каналов с помощью двух затворов.

На рис. 4 приведены зависимости транскондактанса подвешенного КТК от суммы затворных напряжений и напряжения между истоком и стоком при разности затворных напряжений $\pm 4 \mathrm{~B}$. Указанные разности напряжений на боковых затворах соответствуют одноканальным областям. Плато, соответствующие значениям кондактанса $2 e^{2} / h$ и $4 e^{2} / h$, имеют форму ромбов. Вертикальный размер ромбов определяется шириной щели между одномерными подзонами. Из эксперимента следует, что характерная величина межподзонной щели в обоих каналах составляет $\sim 12$ мэВ, что хорошо согласуется с результатами других экспериментов с подвешенными КТК [29,30].

\section{4. Обсуждение результатов}

Зная характерную ширину межподзонной щели $\Delta E \approx 12$ мэВ, можно оценить ширину канала $\Delta x$. Если предположить, что ограничивающий потенциал вблизи дна параболический, то энергия на крае канала определяется выражением

$$
E=m^{*} \omega^{2}(\Delta x / 2)^{2} / 2,
$$

где $\omega=\Delta E / \hbar, m^{*}$ - эффективная масса электрона в GaAs. Оценка, например, для второй подзоны, для которой $E=3 \hbar \omega / 2$, дает $\Delta x \approx 30$ нм, что сравнимо с фермиевской длиной волны электрона в ДЭГ, но в $~ 30$ раз меньше литографической ширины КТК. Таким образом, каналы оказываются достаточно узкими.

Обсудим природу возникновения двух проводящих каналов внутри одиночного КТК. Одна из возможных причин - наличие дополнительных $X$-долинных электронов в сверхрешетках используемых гетероструктур GaAs/AlGaAs. В структурах, в которых таких электронов нет, вытравливание литографических траншей и захват электронов на поверхностные состояния на литографических краях приводят к краевому обеднению ДЭГ и образованию одиночного канала вблизи середины сужения (см. рис. $5, b)$. В структурах же, содержащих $X$-долинные электроны, электростатика существенно иная. В результате вытравливания траншей электроны в $X$-долинах, отталкиваясь от поверхностных электронов, собираются вблизи середины микросужения (см. рис. $5, c)$, формируя в этом месте дополнительный отталкивающий потенциал (барьер) для электронов в ДЭГ. В результате, как видно из рис. 5, , в КТК образуются два канала, разнесенные симметрично относительно середины КТК. Данный эффект усиливается при подвешивании благодаря усилению кулоновского взаимодействия, обусловлен- 

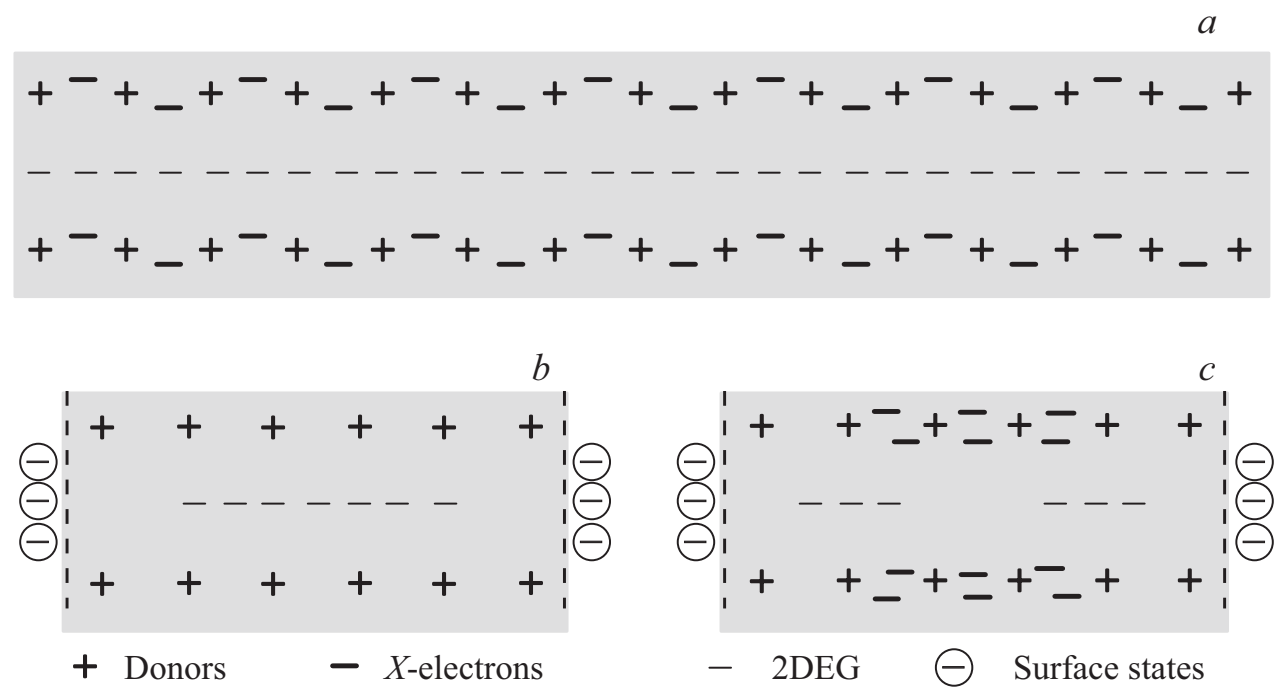

Рис. 5. Схематическое изображение распределения зарядов в гетероструктуре: $a-$ без литографических краев, $b-$ при наличии литографических краев в отсутствие $X$-долинных электронов, $c$ - при наличии литографических краев и $X$-долинных электронов.

ному отрывом КТК от подложки с высокой диэлектрической проницаемостью. Такое объяснение согласуется с вышеописанными экспериментальными зависимостями кондактанса от напряжений на боковых затворах.

\section{5. Заключение}

Экспериментально изучен кондактанс подвешенных, т. е. отсоединенных от подложки, квантовых точечных контактов, изготовленных на основе гетероструктур $\mathrm{GaAs} / \mathrm{AlGaAs}$ с двумерным электронным газом, снабженных латеральными затворами, отделенными от микросужения с помощью литографических траншей. Показано, что электронный транспорт в таких структурах осуществляется в необычном двухканальном режиме с независимым квантованием кондактанса отдельных каналов, управляемым с помощью двух затворов. Возможная причина возникновения двухканальной структуры связана с латеральным перераспределением низкоподвижных $X$-долинных электронов, приводящим к образованию в середине КТК потенциального барьера для электронов в ДЭГ, происходящим в результате вытравливания литографических траншей, задающих геометрию наноструктуры.

\section{Благодарности}

Авторы выражают благодарность ЦКП „ВТАН“ НГУ за предоставленное оборудование для электронной литографии.

\section{Финансирование работы}

Работа выполнена при поддержке РФФИ (грант № 19-02-00800-А, экспериментальное исследование), РНФ (грант № 18-72-10058, изготовление образцов) и Программы фундаментальных исследований ИФП СО РАН (грант № 0306-2019-0019, характеризация образцов).

\section{Конфликт интересов}

Авторы заявляют, что у них нет конфликта интересов.

\section{Список литературы}

[1] B.J. van Wees, H. van Houten, C.W.J. Beenakker, J.G. Williamson, L.P. Kouwenhoven, D. van der Marel, C.T. Foxon. Phys. Rev. Lett., 60, 848 (1988).

[2] D.A. Wharam, T.J. Thornton, R. Newbury, M. Pepper, H. Ahmed, J.E.F. Frost, D.G. Hasko, D.C. Peacock, D.A. Ritchie, G.A.C. Jones. J. Phys. C: Solid State Phys., 21, L209 (1988).

[3] P. Debray, S.M.S. Rahman, J. Wan, R.S. Newrock, M. Cahay, A.T. Ngo, S.E. Ulloa, S.T. Herbert, M. Muhammad, M. Johnson. Nature Nanotechnology, 4, 759 (2009).

[4] D.A. Pokhabov, A.G. Pogosov, E.Yu. Zhdanov, A.A. Shevyrin, A.K. Bakarov, A.A. Shklyaev. Appl. Phys. Lett., 112, 082102 (2018).

[5] T. Masuda, K. Sekine, K. Nagase, K.S. Wickramasinghe, T.D. Mishima, M.B. Santos, Y. Hirayama. Appl. Phys. Lett., 112, 192103 (2018).

[6] D.A. Pokhabov, A.G. Pogosov, E.Yu. Zhdanov, A.K. Bakarov, A.A. Shklyaev. Appl. Phys. Lett., 115, 152101 (2019).

[7] А.Г. Погосов, М.В. Буданцев, Р.А. Лавров, А.Е. Плотников, А.К. Бакаров, А.И. Торопов, Ж.К. Портал. Письма ЖЭТФ, 83, 152 (2006).

[8] А.Г. Погосов, М.В. Буданцев, А.А. Шевырин, А.Е. Плотников, А.К. Бакаров, А.И. Торопов. Письма ЖЭТФ, 87, 176 (2008).

[9] Е.Ю. Жданов, А.Г. Погосов, М.В. Буданцев, Д.А. Похабов, А.К. Бакаров. ФТП, 51, 12 (2017). 
[10] D.A. Pokhabov, A.G. Pogosov, A.A. Shevyrin, E.Yu. Zhdanov, A.K. Bakarov, A.A. Shklyaev, S.V. Ishutkin, M.V. Stepanenko, E.V. Shesterikov. J. Phys.: Conf. Ser., 964, 012008 (2018).

[11] Y. Gindikin, V.A. Sablikov. Phys. Rev. B, 98, 115137 (2018).

[12] Y. Gindikin, V.A. Sablikov. Physica E, 108, 187 (2019).

[13] Y. Gindikin, V.A. Sablikov. Eur. Phys. J. Spec. Top., 229, 503 (2020).

[14] A.G. Pogosov, M.V. Budantsev, E.Yu. Zhdanov, D.A. Pokhabov, A.K. Bakarov, A.I. Toropov. Appl. Phys. Lett., 100, 181902 (2012).

[15] E.Yu. Zhdanov, A.G. Pogosov, M.V. Budantsev, D.A. Pokhabov. AIP Conf. Proc., 1566, 211 (2013).

[16] K.J. Thomas, J.T. Nicholls, M.Y. Simmons, W.R. Tribe, A.G. Davies, M. Pepper. Phys. Rev. B, 59, 12252 (1999).

[17] I.M. Castleton, A.G. Davies, A.R. Hamilton, J.E.F. Frost, M.Y. Simmons, D.A. Ritchie, M. Pepper. Physica B, 249-251, 157 (1998).

[18] L.W. Smith, W.K. Hew, K.J. Thomas, M. Pepper, I. Farrer, D. Anderson, G.A.C. Jones, D.A. Ritchie. Phys. Rev. B, 80, 041306 (2009).

[19] W.K. Hew, K.J. Thomas, M. Pepper, I. Farrer, D. Anderson, G.A.C. Jones, D.A. Ritchie. Physica E, 42, 1118 (2010).

[20] L.W. Smith, W.K. Hew, K.J. Thomas, M. Pepper, I. Farrer, D. Anderson, G.A.C. Jones, D.A. Ritchie. Physica E, 42, 1114 (2010).

[21] S. Kumar, K.J. Thomas, L.W. Smith, M. Pepper, G.L. Creeth, I. Farrer, D. Ritchie, G. Jones, J. Griffiths. Phys. Rev. B, 90, 201304(R) (2014).

[22] А.В. Чаплик. Письма ЖЭТФ, 31, 275 (1980).

[23] J.S. Meyer, K.A. Matveev. J. Phys.: Condens. Matter, 21, 023203 (2009)

[24] S.-C. Ho, H.-J. Chang, C.-H. Chang, S.-T. Lo, G. Creeth, S. Kumar, I. Farrer, D. Ritchie, J. Griffiths, G. Jones, M. Pepper, T.-M. Chen. Phys. Rev. Lett., 121, 106801 (2018).

[25] C. Yan, S. Kumar, K. Thomas, P. See, I. Farrer, D. Ritchie, J. Griffiths, G. Jones, M. Pepper. J. Phys.: Condens. Matter, 30, 08LT01 (2018).

[26] K.-J. Friedland, R. Hey, H. Kostial, R. Klann, K. Ploog. Phys. Rev. Lett., 77, 4616 (1996).

[27] N. Bhandari, P.P. Das, M. Cahay, R.S. Newrock, S. T. Herbert. Appl. Phys. Lett., 101, 102401 (2012).

[28] P.P. Das, N.K. Bhandari, J. Wan, J. Charles, M. Cahay, K.B. Chetry, R.S. Newrock, S.T. Herbert. Nanotechnology, 23, $215201(2012)$

[29] C. Rössler, M. Herz, M. Bichler, S. Ludwig. Solid State Commun., 150, 861 (2010).

[30] A.A. Shevyrin, A.G. Pogosov, M.V. Budantsev, A.K. Bakarov, A.I. Toropov, S.V. Ishutkin, E.V. Shesterikov. Appl. Phys. Lett., 104, 203102 (2014).

Редактор Л.В. Шаронова

\section{Double-channel electron transport in suspended quantum point contacts with in-plane side gates}

\section{D.A. Pokhabov 1,2 , A.G. Pogosov 1,2 , E.Yu. Zhdanov ${ }^{1,2}$,} A.K. Bakarov ${ }^{1,2}$, A.A. Shklyaev ${ }^{1,2}$

${ }^{1}$ Rzhanov Institute of Semiconductor Physics, Siberian Branch of Russian Academy of Sciences, 630090 Novosibirsk, Russia

${ }^{2}$ Novosibirsk State University, 630090 Novosibirsk, Russia

\begin{abstract}
The conductance of a suspended quantum point contact fabricated from a GaAs/AlGaAs heterostructure with a two-dimensional electron gas, equipped with in-plane side gates separated from the constriction using lithographical trenches, is studied. The conductance as a function of the gate voltages demonstrates unusual double-channel regime with independent channel conductance quantization: two side gates can drive the conductance of the separate channels independently. A possible electrostatic mechanism of the double-channel structure formation inside a single construction is connected with the lateral redistribution of the low-mobility $X$-valley electrons contained in superlattice layers, resulting in the emergence of the potential barrier in the middle of the quantum point contact, separating the conducting electrons into two channels, symmetrically shifted towards the lithographical trenches, defining the nanostructure geometry.
\end{abstract}

